\section{Comparación de la profundidad de polimerización de resinas compuestas bulk fill obtenida con dos unidades de fotoactivación LED: polywave versus monowave}

\section{Depth of cure comparison of bulk-fill resin composites with two LED light-curing units: polywave versus monowave}

\begin{abstract}
Resumen
Objetivo. Comparar la profundidad de polimerización de tres resinas compuestas bulk fill obtenida con dos unidades de fotoactivación LED (polywave versus monowave). Métodos. Con las resinas compuestas bulk fill: Filtek One Bulk Fill Restorative (FO) (3M ESPE), Tetric N-Ceram Bulk Fill (TNC) (Ivoclar Vivadent) y Opus Bulk Fill APS (OP) (FGM), fueron confeccionados cuerpos de prueba $(\mathrm{n}=5)$ de formato cilíndrico $(4 \mathrm{~mm}$ diámetro y $10 \mathrm{~mm}$ altura) para la evaluación de la profundidad de polimerización según la norma ISO 4049. Fueron empleadas dos unidades de fotoactivación LED de diferentes tecnologías: Bluephase N (Tecnología Polywave - Ivoclar Vivadent) y LED D (Tecnología Monowave - Woodpecker) con densidad de energía de $24 \mathrm{~J} / \mathrm{cm}^{2}$. Los datos fueron analizados por medio del análisis de la varianza de dos factores y la prueba post hoc de Tukey ( $\alpha$ $=5 \%)$. Resultados. LED D produjo mayores valores de profundidad de polimerización en todas las resinas compuestas bulk fill evaluadas $(\mathrm{p}<0,05)$. Los valores obtenidos con Bluephase N fueron: 3,87 (TNC) > 3,74 (FO) > 3,31 (OP) y con LED D fueron 4,29 (TNC) > 4,11 (FO) > 3,92 (OP). Conclusiones. La unidad de fotoactivación LED de tecnología monowave produjo mayor profundidad de polimerización que la de tecnología polywave, independientemente de la resina compuesta bulk fill utilizada. Las resinas compuestas tuvieron diferentes valores de profundidad de polimerización.
\end{abstract}

Palabras clave: Resinas compuestas; Polimerización; Propiedades físicas y químicas; Materiales dentales; Odontología (fuente: DeCS BIREME).

\footnotetext{
Abstract

Objective. To compare the depth of cure of three bulk-fill resin composites using polywave vs. monowave LED light-curing units. Methods. Cylindrical specimens (4 $\mathrm{mm}$ diameter and $10 \mathrm{~mm}$ height) were prepared using resin composites: Filtek One Bulk Fill Restorative (FO) (3M ESPE), Tetric N-Ceram Bulk Fill (TNC) (Ivoclar Vivadent) and Opus Bulk Fill APS (OP) (FGM), to evaluate the depth of cure according to ISO 4049 standard $(\mathrm{n}=5)$. Two LED light-curing units were used: Bluephase $\mathrm{N}$ (Polywave, Ivoclar Vivadent) and LED D (Monowave, Woodpecker) with $24 \mathrm{~J} / \mathrm{cm}^{2}$ of energy density. Data were analyzed by two-way Analysis of Variance and Tukey's post
}

Artículo Original

Allan Gutierrez-Leiva ${ }^{1, a}$, César Pomacóndor-Hernández ${ }^{2}$

1 Universidad Nacional Mayor de San Marcos, Facultad de Odontología, Lima, Perú.

${ }^{2}$ Universidad Privada San Juan Bautista, Escuela de

Estomatología, Lima, Perú.

${ }^{a}$ Cirujano Dentista.

${ }^{b}$ Doctor en Materiales Dentales.

Correspondencia:

Allan Gutierrez-Leiva: allangutierrezleiva@gmail.com Calle 39, Mz. I-5, Lt. 26, Urbanización Pro $2^{\mathrm{a}}$ Etapa, Los

Olivos. Lima, Perú.

ORCID: 0000-0001-8758-3349

\section{Coautores:}

César Pomacóndor-Hernández: cesar.pomacondor. hernandez@gmail.com

ORCID: 0000-0001-9951-4797

Editora:

Rosse Mary Falcón-Antenucci

Universidad Inca Garcilaso de la Vega, Perú.

Conflicto de intereses: los autores declaran no tener conflicto de intereses

Financiamiento: este estudio fue financiado con recursos propios de los autores.

Recibido: $16 / 01 / 20$

Aceptado: 16/03/20

Publicado: 09/05/20 
hoc test $(\alpha=5 \%)$. Results. LED D group showed higher depth of cure values in all resin composites evaluated $(\mathrm{p}<0.05)$. The values were: Bluephase $\mathrm{N}: 3.87$ (TNC) > $3.74(\mathrm{FO})>3.31(\mathrm{OP})$, and LED D: $4.29(\mathrm{TNC})>4.11(\mathrm{FO})>3.92(\mathrm{OP})$. Conclusions. The monowave LED light-curing unit produced greater depth of cure than the polywave, regardless of the bulk-fill resin composite used. Resin composites showed different depth of cure values.

Keywords: Composite resins; Polymerization; Physicochemical phenomena; Dental materials; Dentistry (source: MeSH NLM).

\section{Introducción}

La resina compuesta es el material restaurador directo más empleado en Odontología debido a la capacidad de adherirse al tejido dental, requerir una técnica poco invasiva, y a sus excelentes características estéticas. Sin embargo, presenta limitaciones que pueden afectar la longevidad de las restauraciones, y contra las cuales los fabricantes continuamente buscan estrategias para reducirlas ${ }^{1,2}$. Una de estas limitaciones ocurre durante el proceso de polimerización en el que la resina compuesta sufre inevitablemente contracción dimensional, la cual genera tensiones en las paredes de la cavidad, y tienden a producir flexión cuspídea, microfracturas de esmalte y pérdida de la integridad de la interfase adhesiva ${ }^{3}$. Además, si la resina compuesta aplicada es de gran espesor, la penetración de la luz de fotoactivación disminuye causando una polimerización defectuosa ${ }^{4,5}$.

La técnica incremental se ha utilizado para reducir las tensiones generadas por la contracción de polimerización y garantizar una adecuada penetración de la luz activadora. Esta técnica establece la inserción de incrementos de resina compuesta en un espesor máximo de 2 $\mathrm{mm}$, los cuales deben ser fotoactivados individualmente 6,7. Sin embargo, algunos estudios recientes indican que la técnica incremental podría causar aún mayores efectos perjudiciales de tensión por contracción de polimerización que una técnica monoincremental ${ }^{8,9}$. Además, la inserción de varios incrementos también presenta inconvenientes como son el mayor tiempo empleado por el profesional para restaurar cavidades profundas, el atrapamiento de aire y la posibilidad de contaminación entre los incrementos de resina ${ }^{3,6}$.

Las innovaciones en el desarrollo de resinas compuestas están orientadas a superar las limitaciones previamente descritas del material y reducir el tiempo clínico operatorio. Recientemente han hecho su aparición en el mercado las resinas compuestas monoincrementales más conocidas como resinas compuestas bulk fill. Este nuevo material tiene como principal ventaja el permitir la aplicación de incrementos de $4-5 \mathrm{~mm}$ con una única irradiación de la luz de fotoactivación para todo ese bloque ${ }^{10}$. De esa forma, el tiempo clínico es reducido ${ }^{11}$, se genera menor tensión por contracción, y se garantiza una adecuada polimerización en todo el monoincremento $5,12,13$. Estas características convierten a las resinas compuestas bulk fill en el material de elección para realizar de forma simplificada las restauraciones dentales de gran tamaño, sobre todo las del sector posterior ${ }^{10}$.
Para el alivio de las tensiones generadas y el incremento de la profundidad de polimerización se realizaron algunas modificaciones en la composición química de las resinas compuestas, como el aumento de la translucidez para una mayor transmisión de la luz activadora, así como la incorporación de monómeros moduladores de la polimerización y la modificación de partículas de relleno ${ }^{14,15}$. Asimismo, a pesar que la mayoría de resinas bulk fill utiliza, al igual que las resinas convencionales, la canforoquinona como fotoiniciador (espectro de absorción: $390-510 \mathrm{~nm}$ ) y la amina terciaria como coiniciador, otros fotoiniciadores alternativos también pueden ser incorporados. Por ejemplo, la empresa Ivoclar Vivadent utiliza, además de la canforoquinona, un óxido de acilfosfina (Lucirin TPO - espectro de absorción: 300 $-420 \mathrm{~nm}$ ) y un derivado del dibenzoilo de germanio (patentado como Ivocerin - espectro de absorción: 370 $-460 \mathrm{~nm}$ ) para asegurar la adecuada polimerización de grandes incrementos ${ }^{16,17}$.

Para activar los fotoiniciadores de las resinas compuestas actualmente se emplean las unidades de fotoactivación LED (light emitting diode). La canforoquinona, principal fotoiniciador de los materiales dentales resinosos, necesita una longitud de onda ideal de 420 - $495 \mathrm{~nm}$ para activarse, mientras que los fotoiniciadores alternativos por lo general presentan un espectro de absorción de luz en longitud de onda menor de $420 \mathrm{~nm}$. Debido a esta diferencia de espectros ideales para cada tipo de fotoiniciador existen 2 tecnologías distintas de unidades de fotoactivación LED: monowave y polywave. Las primeras poseen diodos emisores sólo en el rango de luz azul (420 - 495nm), adecuado para la activación de la canforoquinona, mientras que las segundas poseen, además del rango azul, diodos emisores en el espectro violeta $(380-420 \mathrm{~nm})$ para activar los fotoiniciadores alternativos ${ }^{18,19}$.

Actualmente no existe un consenso sobre la necesidad del uso de una unidad de fotoactivación LED polywave para obtener la polimerización óptima de las resinas compuestas bulk fill. Esta interrogante cobra aún mayor importancia porque la mayoría de odontólogos utilizan unidades de fotoactivación monowave debido a que existe una mayor oferta en el mercado y son más accesibles económicamente. Además, recientemente fueron lanzadas nuevas resinas compuestas bulk fill y aún existe escasa información sobre la profundidad de polimerización que pueden alcanzar con diferentes unidades fotoactivadoras. Por lo tanto, el objetivo del presente estudio fue comparar la profundidad de 
polimerización de tres productos comerciales de resina compuesta bulk fill obtenida con dos unidades de fotoactivación LED de diferente tecnología: polywave versus monowave.

\section{Métodos}

Diseńo experimental. En el presente estudio in vitro de enfoque cuantitativo se evaluó la variable dependiente profundidad de polimerización y las variables independientes: a) Producto comercial de resina compuesta bulk fill y b) Tecnología de unidad de fotoactivación LED. Fueron utilizadas tres resinas compuestas bulk fill: Filtek One Bulk Fill Restorative (FO), Tetric N-Ceram Bulk Fill (TNC) y Opus Bulk Fill APS (OP) empleando dos unidades de fotoactivación LED: Bluephase N (de tecnología polywave) y LED D (de tecnología monowave). Los nombres de los fabricantes y otras informaciones sobre las resinas compuestas bulk fill y las unidades de fotoactivación LED utilizadas en este estudio están descritas en la Tabla 1 y 2 , respectivamente.

Cálculo de los tiempos de fotoactivación. La potencia de las unidades de fotoactivación LED fue medida antes y durante el estudio utilizando el radiómetro Bluephase Meter II (Ivoclar Vivadent, Schaan, Liechtenstein). Para obtener la irradiancia, los valores de potencia fueron divididos entre el área de la punta de cada unidad de fotoactivación LED (Tabla 2). Debido a la diferencia marcada entre las irradiancias obtenidas por Bluephase $\mathrm{N}\left(1177 \mathrm{~mW} / \mathrm{cm}^{2}\right)$ y LED D $\left(826 \mathrm{~mW} / \mathrm{cm}^{2}\right)$, fue necesario estandarizar la densidad de energía que recibirían los cuerpos de prueba de resina compuesta bulk fill utilizando la fórmula ${ }^{20}$ :

$$
\begin{gathered}
\text { Irradiancia }\left(\mathrm{mW} / \mathrm{cm}^{2}\right) \times \text { Tiempo }(s)=\text { Densidad de } \\
\text { energía }\left(\mathrm{J} / \mathrm{cm}^{2}\right)
\end{gathered}
$$

De esa manera, los tiempos de fotoactivación utilizados con Bluephase N y LED D fueron de 20 s y 30 s, respectivamente, para estandarizar una densidad de energía aproximada de $24 \mathrm{~J} / \mathrm{cm}^{2}$ sobre el material.

Profundidad de polimerización. La metodología fue realizada en un ambiente con temperatura controlada $\left(23^{\circ} \mathrm{C}\right)$ y utilizando iluminación artificial de color rojo (por ser distante al espectro azul y violeta) para evitar posibles interferencias en la polimerización de las resinas. Fueron confeccionados un total de 30 cuerpos de prueba de resina compuesta bulk fill $(\mathrm{n}=5)$ utilizando una matriz metálica de acero inoxidable en formato cilíndrico de 4 $\mathrm{mm}$ de diámetro y $10 \mathrm{~mm}$ de altura (Figura 1), como está

\begin{tabular}{|c|c|c|c|c|}
\hline $\begin{array}{l}\text { Producto comercial } \\
\text { (Fabricante) }\end{array}$ & Tonalidad & Composición & $\begin{array}{l}\text { Máximo espesor } \\
\text { recomendado }\end{array}$ & $\begin{array}{l}\text { Tiempo de fotoactivación } \\
\text { recomendado }\end{array}$ \\
\hline $\begin{array}{l}\text { Filtek One Bulk Fill Restorative - FO } \\
\text { (3M ESPE, St. Paul, EEUU) }\end{array}$ & A2 & $\begin{array}{l}\text { AUDMA, AFM, DDDMA, } \\
\text { UDMA, trifluoruro de iterbio, } \\
\text { nanopartículas de sílice, } \\
\text { nanopartículas de zirconia, } \\
\text { nanoaglomerados de sílice/ } \\
\text { zirconia }\end{array}$ & $4 \mathrm{~mm}$ & $\begin{array}{l}1000-2000 \mathrm{~mW} / \mathrm{cm}^{2}: 20 \mathrm{~s} \\
550-1000 \mathrm{~mW} / \mathrm{cm}^{2}: 40 \mathrm{~s}\end{array}$ \\
\hline $\begin{array}{l}\text { Tetric N-Ceram Bulk Fill - TNC } \\
\text { (Ivoclar Vivadent, Schaan, Liech- } \\
\text { tenstein) }\end{array}$ & IVA & $\begin{array}{l}\text { Dimetacrilatos, vidrio de } \\
\text { bario, prepolímero, trifluoruro } \\
\text { de iterbio, óxido mixto, aditi- } \\
\text { vos, catalizadores, estabiliza- } \\
\text { dores y pigmentos }\end{array}$ & $4 \mathrm{~mm}$ & $\begin{array}{l}\geq 1000 \mathrm{~mW} / \mathrm{cm}^{2}: 10 \mathrm{~s} \\
\geq 500 \mathrm{~mW} / \mathrm{cm}^{2}: 20 \mathrm{~s}\end{array}$ \\
\hline $\begin{array}{l}\text { Opus Bulk Fill APS - OP } \\
\text { (FGM, Joinville, Brasil) }\end{array}$ & $\mathrm{A} 2$ & $\begin{array}{l}\text { Monómeros uretanadimeta- } \\
\text { crílicos, fotoiniciadores (APS), } \\
\text { coiniciador, sílice silanizado, } \\
\text { estabilizantes, pigmentos }\end{array}$ & $5 \mathrm{~mm}$ & $\begin{array}{l}1000-2000 \mathrm{~mW} / \mathrm{cm}^{2}: 30 \mathrm{~s} \\
750-1000 \mathrm{~mW} / \mathrm{cm}^{2}: 40 \mathrm{~s}\end{array}$ \\
\hline
\end{tabular}

Tabla 1. Información de las resinas compuestas bulk fill empleadas en este estudio

AUDMA: dimetacrilato de uretano aromático; AFM, monómeros de adición - fragmentación; DDDMA: 1, 12-dimetacrilato dodecanodiol; UDMA: dimetacrilato de uretano

\begin{tabular}{|c|c|c|c|c|}
\hline $\begin{array}{c}\text { LED } \\
\text { (Fabricante) }\end{array}$ & $\begin{array}{c}\text { Tecnología / Longitud de onda / } \\
\text { Irradiancia* }\end{array}$ & $\begin{array}{l}\text { Diámetro activo de la } \\
\text { punta** }\end{array}$ & Potencia medida & Irradiancia calculada \\
\hline $\begin{array}{l}\text { Bluephase N } \\
\text { (Ivoclar Vivadent, Schaan, } \\
\text { Liechtenstein) }\end{array}$ & $\begin{array}{c}\text { Polywave } \\
385-515 \mathrm{~nm} \\
1200 \mathrm{~mW} / \mathrm{cm}^{2}\end{array}$ & $8,8 \mathrm{~mm}$ & $716 \mathrm{~mW}$ & $1177 \mathrm{~mW} / \mathrm{cm}^{2}$ \\
\hline $\begin{array}{l}\text { LED D } \\
\text { (Woodpecker, Guilin, China) }\end{array}$ & $\begin{array}{c}\text { Monowave } \\
420-480 \mathrm{~nm} \\
850-1000 \mathrm{~mW} / \mathrm{cm}^{2}\end{array}$ & $6,8 \mathrm{~mm}$ & $300 \mathrm{~mW}$ & $826 \mathrm{~mW} / \mathrm{cm}^{2}$ \\
\hline
\end{tabular}

Tabla 2. Información de las unidades de fotoactivación LED empleadas en este estudio

\footnotetext{
* Datos proporcionados por los fabricantes

** Se desconsideró $1 \mathrm{~mm}$ correspondiente al borde oscuro que carece de fibra óptica
} 
descrito en las especificaciones de la norma ISO 4049 sobre profundidad de polimerización ${ }^{21}$. Considerando que la matriz metálica consiste en un agujero cilíndrico, ésta fue siempre posicionada sobre un fondo blanco para estandarizar la reflexión/absorción de la luz emitida por la unidad de fotoactivación LED. Así, una lámina portaobjetos fue colocada sobre la superficie blanca y fue cubierta por una cinta matriz de poliéster. La matriz metálica se posicionó sobre la cinta matriz de poliéster y a continuación, usando una espátula para inserción, se empaquetó en su interior la resina compuesta bulk fill hasta llenarla por completo. Inmediatamente otra cinta matriz de poliéster seguida por otra lámina portaobjetos fueron colocadas en la superficie de la matriz metálica ejerciendo presión manual sobre ésta para eliminar los excesos de material. Se procedió a realizar la fotoactivación con la punta de salida de luz de la LED en íntimo contacto con la lámina portaobjetos, la cual no fue removida como indica la ISO 4049 para poder simular la distancia aproximada de $1 \mathrm{~mm}$ que existe en una situación clínica entre la punta de salida de luz de la
LED y el material restaurador (Figura 2). Después de la fotoactivación, los cuerpos de prueba cilíndricos fueron retirados de la matriz metálica (Figura 3) y se eliminó el material no polimerizado en la parte inferior mediante raspado con espátula de plástico (Figura 4). La longitud resultante del cuerpo de prueba se midió con un calibrador digital (de sensibilidad 0,01 $\mathrm{mm}$ ) en cinco puntos diferentes registrándose el promedio como la longitud final (Figura 5). Este valor se dividió entre dos para obtener así el valor de la profundidad de polimerización de cada cuerpo de prueba ${ }^{21}$.

Análisis estadístico. Los valores de profundidad de polimerización fueron analizados por medio del análisis de la varianza de dos factores y la prueba post hoc de Tukey $(\alpha=5 \%)$ empleando el programa estadístico SigmaPlot 12,0 (Systat Software Inc., San Jose, USA).

\section{Resultados}

Los promedios de los valores de profundidad de polimerización y sus desviaciones estándar están descritos en

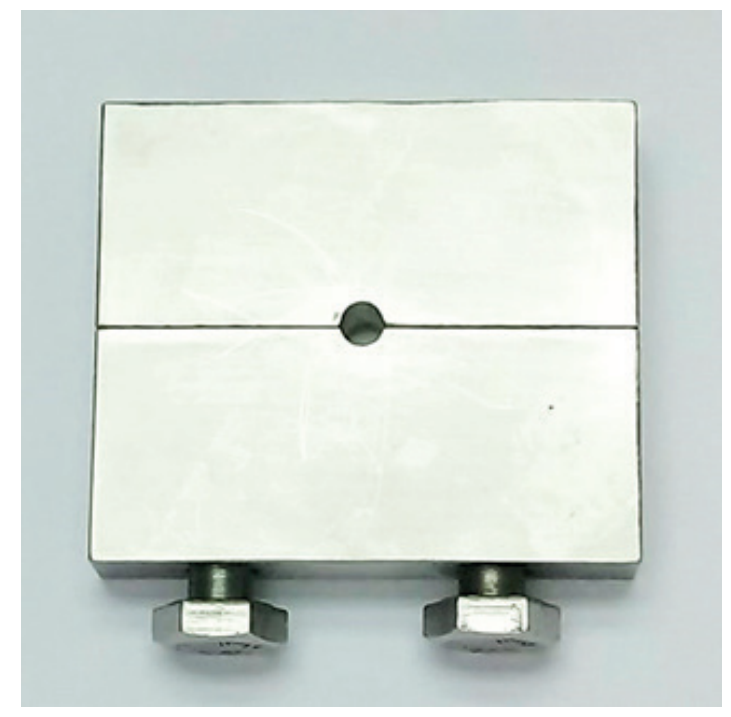

Figura 1. Matriz metálica de acero inoxidable usada para evaluación de la profundidad de polimerización según la norma ISO 4049.

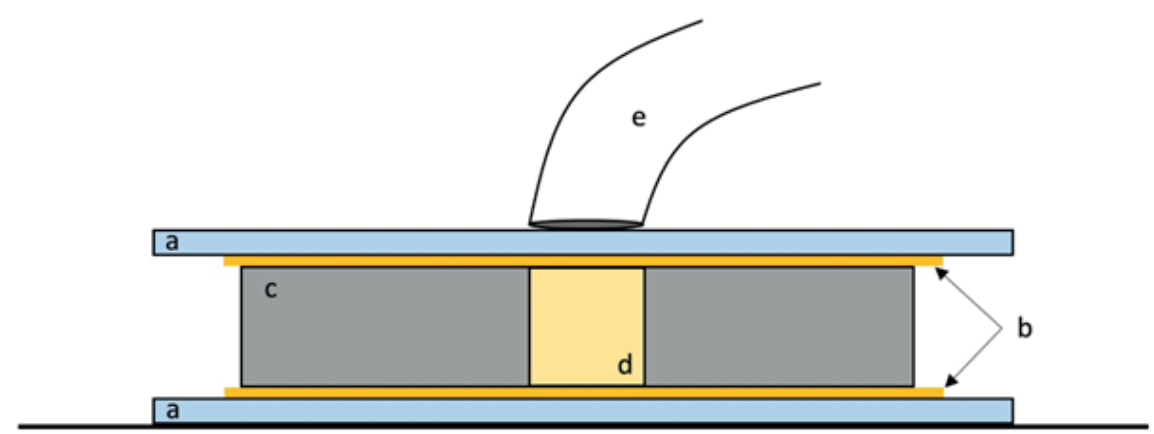

Figura 2. Esquema que ejemplifica la elaboración de los cuerpos de prueba. a: lámina portaobjetos de $1 \mathrm{~mm}$ de espesor; $b$ : cinta matriz de poliéster; c: matriz metálica de acero inoxidable en formato cilíndrico de $4 \mathrm{~mm}$ de diámetro y $10 \mathrm{~mm}$ de altura; $\mathrm{d}$ : cuerpo de prueba de resina compuesta bulk fill; e: punta de salida de luz de la unidad de fotoactivación LED 


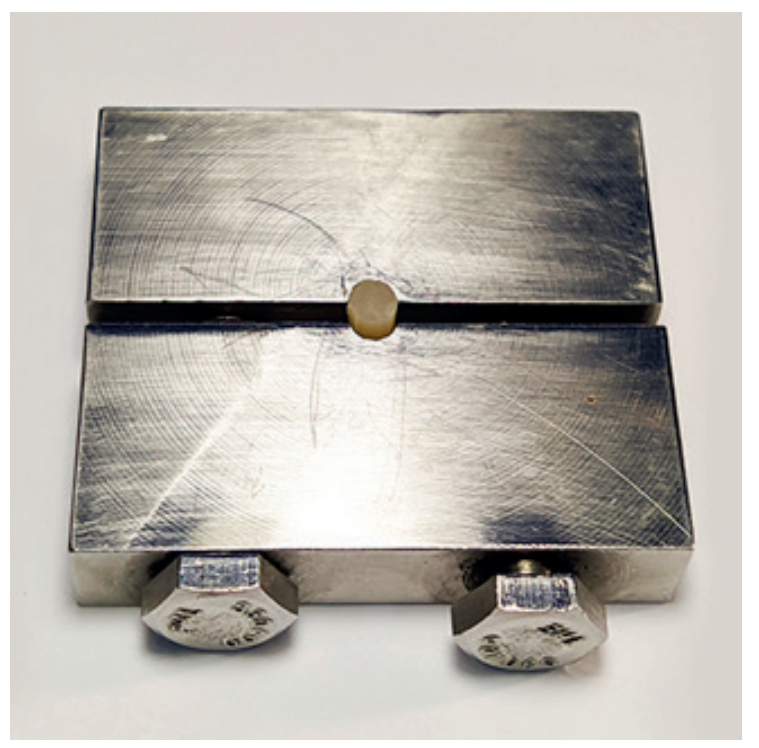

Figura 3. Remoción del cuerpo de prueba de resina compuesta bulk fill de la matriz metálica

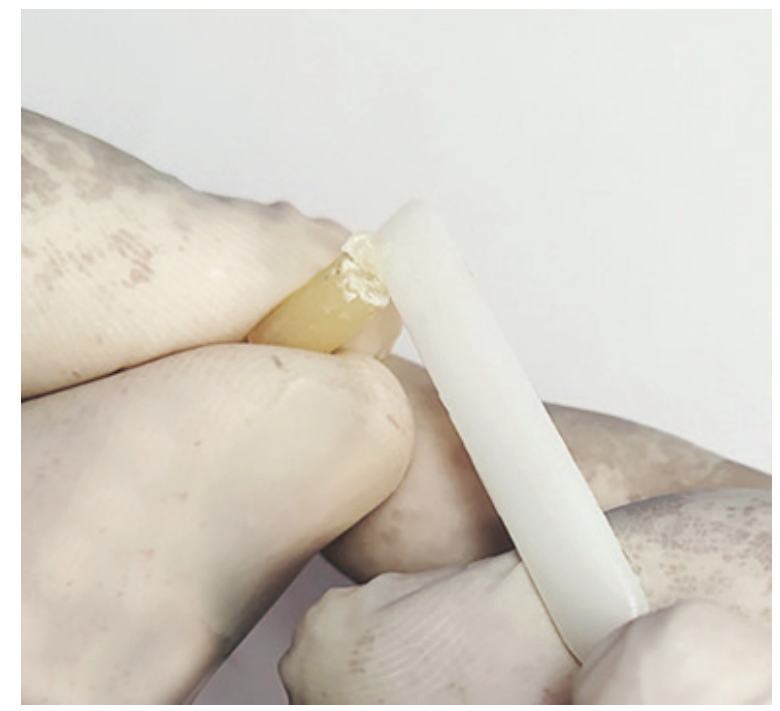

Figura 4. Raspado con espátula de plástico para eliminar el material no polimerizado de la parte inferior del cuerpo de prueba

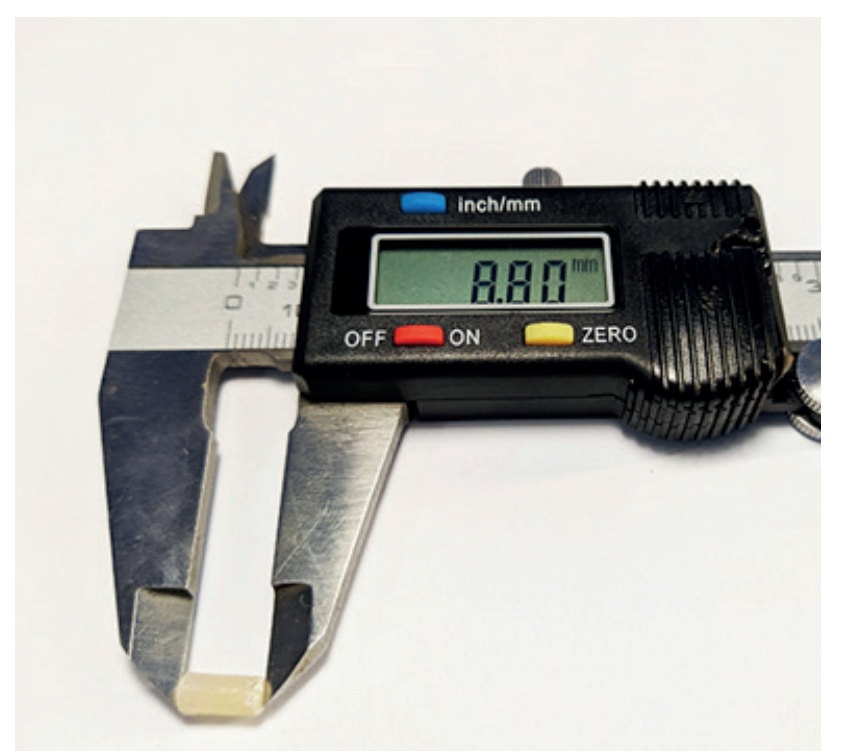

Figura 5. Medida de la longitud del cuerpo de prueba obtenida después del raspado 
la Tabla 3. El análisis estadístico mostró que Bluephase $\mathrm{N}$ produjo menores valores de profundidad de polimerización que los obtenidos con LED D $(\mathrm{p}<0,05)$ en todas las resinas compuestas bulk fill estudiadas. Con ambas unidades de fotoactivación LED los valores de profundidad de polimerización fueron estadísticamente diferentes para las tres resinas compuestas bulk fill: TNC $>\mathrm{FO}>\mathrm{OP}(\mathrm{p}<0,05)$.

\section{Discusión}

La determinación de la profundidad de polimerización de una resina compuesta busca identificar el espesor máximo en el que esta puede ser aplicada y fotoactivada sobre el diente garantizando conservar adecuadas propiedades mecánicas y biocompatibilidad ${ }^{22}$. La profundidad de polimerización, también conocida como profundidad de curado, es dependiente de la composición química y propiedades físicas del material, así como de los diversos factores que participan en el proceso de fotoactivación ${ }^{22}$. En este estudio in vitro se evaluó la profundidad de polimerización de tres productos comerciales de resina compuesta bulk fill utilizando dos unidades de fotoactivación LED de diferente tecnología (polywave vs. monowave) obteniéndose que la unidad monowave produjo mayores valores de profundidad de polimerización que la polywave, independientemente de la resina compuesta bulk fill utilizada ( $<<0,05)$ (Tabla 3).

Existen varios métodos para medir la profundidad de polimerización de una resina compuesta, entre los cuales los más utilizados son: 1) la metodología establecida por las especificaciones de la norma ISO 4049,2) el método por medio de la microdureza, y 3 ) la prueba a través del grado de conversión del material ${ }^{4}$. Si bien es cierto los dos últimos métodos son más precisos en la medición de la profundidad de polimerización, el presente estudio seleccionó la metodología planteada por la ISO 4049 porque es la prueba obligatoria para que los fabricantes puedan certificar y recomendar tiempos de fotoactivación relacionados al espesor del incremento de las resinas compuestas ${ }^{4,23}$, representa una manera práctica de verificar visualmente los resultados del experimento, no requiere de equipos sofisticados, es fácilmente repetible, y sirve para realizar comparaciones de forma simple entre diversos materiales y protocolos de fotoactivación.

Los resultados encontrados en el presente estudio guardan relación con lo descrito recientemente por Gan et al. ${ }^{20}$, quienes por medio de la evaluación del radio de dureza (hardness ratio) de dos resinas compuestas bulk fill concluyeron que utilizando una LED monowave se obtiene mayor profundidad de polimerización que con una LED polywave (estadísticamente significante sólo para la resina compuesta a base de canforoquinona y sin fotoiniciadores alternativos). Otros estudios que utilizaron la metodología de la ISO 4049 y la evaluación por medio del grado de conversión no encontraron diferencias significativas al usar unidades de fotoactivación LED de diferentes tecnologías reforzando la idea que una LED polywave no es indispensable para conseguir mayor profundidad de polimerización en resinas compuestas bulk fill con fotoiniciadores alternativos ${ }^{24-26}$.

El mejor desempeño de la LED monowave en la profundidad de polimerización de las resinas FO y OP, a base de canforoquinona, se puede explicar debido a que estas unidades de fotoactivación emiten un estrecho rango de longitud de onda (420 - $480 \mathrm{~nm}$ ) cuyo pico de espectro de emisión es próximo al pico de absorción de la canforoquinona $(468 \mathrm{~nm})^{25,26}$. Asimismo, la totalidad de densidad de energía emitida por la LED monowave utilizada en el presente estudio $\left(24 \mathrm{~J} / \mathrm{cm}^{2}\right)$ estuvo en el espectro azul. Contrariamente, la LED polywave funciona con diodos emisores azul y violeta, y en consecuencia la densidad de energía emitida no es totalmente del espectro azul requerido por la canforoquinona. Menees et al. ${ }^{24}$ analizaron una LED polywave de similares características a la utilizada en el presente estudio (Bluephase G2 - Ivoclar Vivadent) y relataron que la densidad de energía para cada espectro azul y violeta es de $89 \%$ y $11 \%$ del total, respectivamente.

Una situación diferente se presentó con la resina TNC, que además de la canforoquinona cuenta con fotoiniciadores alternativos como un óxido de acilfosfina (Lucirin TPO) y un derivado del dibenzoilo de germanio (Ivocerin), y podría esperarse un mejor desempeño de la LED polywave ${ }^{17}$. Sin embargo, esto no sucedió probablemente debido a que las unidades de fotoactivación LED polywave presentan baja densidad de energía en el espectro violeta ${ }^{24}$. Adicionalmente, la alta absorción o reactividad de los fotoiniciadores alternativos podría consumir en la superficie la mayor parte de los fotones en el rango violeta, previniendo que lleguen a partes más profundas ${ }^{27}$. También se ha demostrado que, en resinas con fotoiniciador alternativo, las LED polywave aumentan el grado de conversión en la parte superficial, sin embargo, más allá de $2,5 \mathrm{~mm}$ de profundidad

Tabla 3. Promedios (desviación estándar) de la profundidad de polimerización $(\mathrm{mm}$ ) de las resinas compuestas bulk fill según unidad de fotoactivación LED empleada

\begin{tabular}{ccc}
\hline $\begin{array}{c}\text { Producto comercial de resina compuesta } \\
\text { bulk fill }\end{array}$ & $\begin{array}{c}\text { Bluephase } \\
\text { (Tecnología polywave) }\end{array}$ & $\begin{array}{c}\text { LED D } \\
\text { (Tecnología monowave) }\end{array}$ \\
\hline FO & $3,74(0,07) \mathrm{bB}$ & $4,11(0,04) \mathrm{bA}$ \\
TNC & $3,87(0,02) \mathrm{aB}$ & $4,29(0,10) \mathrm{aA}$ \\
OP & $3,31(0,13) \mathrm{cB}$ & $3,92(0,05) \mathrm{cA}$ \\
\hline
\end{tabular}

Diferentes letras minúsculas indican que existen diferencias significativas entre resinas compuestas bulk fill $(p<0,05)$.

Diferentes letras mayúsculas indican que existen diferencias significativas entre unidades LED $(p<0,05)$. 
ya no existe diferencia significativa comparada con una monowave ${ }^{26}$.

Las resinas compuestas bulk fill estudiadas presentaron diferente profundidad de polimerización, independientemente de la unidad de fotoactivación utilizada (TNC $>\mathrm{FO}>\mathrm{OP}$ ) (Tabla 3) debido a la diferente composición química y propiedades físicas ya que el protocolo de fotoactivación fue estandarizado para los tres materiales. La mayor profundidad de polimerización obtenida con la resina TNC se puede atribuir a que presenta la ventaja de tener el fotoiniciador alternativo Ivocerin, el cual absorbe la longitud de onda de luz en un rango más amplio $(370-460 \mathrm{~nm})$. Este fotoiniciador posee un pico de absorción a $408 \mathrm{~nm}$, pero aproximadamente el 50\% se absorbe a $440 \mathrm{~nm}$ localizado en el espectro azul ${ }^{24}$. Además, esta resina presenta alta translucidez que permite una adecuada transmisión de luz activadora a través de ella, facilitando su llegada a regiones más profundas ${ }^{25}$.

Por otro lado, la empresa 3M ESPE afirma que la resina FO trabaja con su propio sistema patentado llamado "Manejo Inteligente del Radio de Contraste" (Smart Contrast Ratio Management), el cual controla la interacción y el índice de refracción entre el componente resinoso y las partículas de relleno, aumentando la opacidad del material durante la fotoactivación ${ }^{28}$. Esto quiere decir que el material es más translúcido antes de ser fotoactivado, permitiendo el paso de la luz a regiones más profundas para alcanzar una adecuada profundidad de polimerización, y durante la fotoactivación cambia el radio de contraste del material tornándolo más opaco y de esa forma mucho más estético ${ }^{28}$. Esto puede explicar los valores muy próximos entre TNC y FO que, a pesar de ser estadísticamente diferentes, la diferencia fue sólo de $0,13 \mathrm{~mm}$ (LED polywave) y 0,18 $\mathrm{mm}$ (LED monowave).

La resina OP obtuvo los valores menores de profundidad de polimerización y no llegó a superar con ninguna de las unidades de fotoactivación LED los $5 \mathrm{~mm}$ de máximo espesor recomendado por el fabricante ${ }^{29}$. Este material funciona con una nueva tecnología APS ( $A d-$ vanced Polymerization System) que reduce la cantidad de canforoquinona en el material al incorporar otros tipos de iniciadores y coiniciadores que son secreto de la marca y que no requieren activación por luz en el espectro violeta ${ }^{30}$. Es relevante remarcar que el tiempo de fotoactivación utilizado en este estudio fue 10 segundos menor a lo recomendado para OP, y esto podría explicar los bajos resultados ${ }^{29}$. Los tiempos de fotoactivación recomendados por cada fabricante son: con Bluephase $\mathrm{N}$ $\left(1177 \mathrm{~mW} / \mathrm{cm}^{2}\right)$ : TNC $-10 \mathrm{~s}, \mathrm{FO}-20$ s y OP $-30 \mathrm{~s}$; con LED D $\left(826 \mathrm{~mW} / \mathrm{cm}^{2}\right)$ : TNC - 20s, FO - 40s y OP - 40s (Tabla 1).

Es importante resaltar que el empleo de la matriz metálica de acero inoxidable para confeccionar los cuerpos de prueba, tal como lo indica la ISO 4049, podría subestimar la profundidad de polimerización que realmente sucede en una situación clínica, debido a que las paredes internas de la matriz metálica no dispersan la luz y por el contrario la absorben, reduciendo la cantidad de fotones disponibles para la activación. La estructura dental no es un cuerpo opaco y la luz que incide alrededor de la resina compuesta puede contribuir con la polimerización gracias a la dispersión de la luz a través de la dentina ${ }^{4}$. Asimismo, se ha demostrado que cuando se usa una matriz a base de teflón de color blanco la profundidad de polimerización es mayor que la obtenida con una matriz metálica de acero inoxidable ${ }^{25}$.

El conocimiento de las propiedades de los materiales es importante para asegurar el éxito de las restauraciones. Según los resultados de este estudio, las unidades de fotoactivación LED monowave logran una adecuada profundidad de polimerización de las resinas compuestas bulk fill, sin embargo, se necesitan más estudios con otras marcas de LED y de resinas compuestas para el mejor entendimiento de esta propiedad. Asimismo, otras metodologías de evaluación de la profundidad de polimerización deben ser utilizadas. A pesar que los resultados refuerzan la idea que no es necesario emplear unidades de fotoactivación LED polywave para lograr una adecuada profundidad de polimerización de resinas compuestas bulk fill con fotoiniciadores alternativos, otras propiedades del material podrían requerir el espectro violeta, por lo tanto, los resultados deben ser tomados con cautela.

Se puede concluir que la unidad de fotoactivación LED de tecnología monowave produjo mayor profundidad de polimerización que la de tecnología polywave, independientemente de la resina compuesta bulk fill utilizada. La profundidad de polimerización varió según el producto comercial (TNC > FO > OP).

\section{Agradecimientos}

A la Universidad Privada Juan Pablo II por posibilitar el uso de sus equipos y laboratorios.

\section{Referencias bibliográficas}

1. Ferracane JL. Resin composite--State of the art. Dent Mater. 2011;27(1):29-38.

2. Pfeifer CS. Polymer-based direct filling materials. Dent Clin North Am. 2017;61(4):733-50.

3. Soares CJ, Faria-E-Silva AL, Rodrigues MP, Vilela ABF, Pfeifer CS, Tantbirojn D, et al. Polymerization shrinkage stress of composite resins and resin cements - What do we need to know? Braz Oral Res. 2017;31(suppl 1):e62.

4. Erickson RL, Barkmeier WW. Comparisons of ISO depth of cure for a resin composite in stainless-steel and natural-tooth molds. Eur J Oral Sci. 2019;127(6):55663.

5. Rizzante FAP, Duque JA, Duarte MAH, Mondelli RFL, Mendonça G, Ishikiriama SK. Polymerization shrinkage, microhardness and depth of cure of bulk fill resin composites. Dent Mater J. 2019;38(3):403-10.

6. Park J, Chang J, Ferracane J, Lee IB. How should composite be layered to reduce shrinkage stress: incremental or bulk filling? Dent Mater. 2008;24(11):1501-5.

7. Chandrasekhar V, Rudrapati L, Badami V, Tummala M. Incremental techniques in direct composite restoration. J Conserv Dent. 2017;20(6):386-91. 
8. Bicalho AA, Valdívia AD, Barreto BC, Tantbirojn D, Versluis A, Soares CJ. Incremental filling technique and composite material--part II: shrinkage and shrinkage stresses. Oper Dent. 2014;39(2):E83-92.

9. Lins RBE, Aristilde S, Osório JH, Cordeiro CMB, Yanikian CRF, Bicalho AA, et al. Biomechanical behaviour of bulk-fill resin composites in class II restorations. J Mech Behav Biomed Mater. 2019;98:255-61.

10. Chesterman J, Jowett A, Gallacher A, Nixon P. Bulk-fill resin-based composite restorative materials: a review. $\mathrm{Br}$ Dent J. 2017;222(5):337-44.

11. Katona A, Barrack I. Comparison of composite restoration techniques. INDECS. 2016;14(1):101-15.

12. Cidreira Boaro LC, Pereira Lopes D, de Souza ASC, Lie Nakano E, Ayala Perez MD, Pfeifer CS, et al. Clinical performance and chemical-physical properties of bulk fill composites resin - a systematic review and meta-analysis. Dent Mater. 2019;35(10):e249-64.

13. Lima RBW, Troconis CCM, Moreno MBP, Murillo-Gómez F, De Goes MF. Depth of cure of bulk fill resin composites: A systematic review. J Esthet Restor Dent. 2018;30(6):492-501.

14. Zorzin J, Maier E, Harre S, Fey T, Belli R, Lohbauer U, et al. Bulk-fill resin composites: polymerization properties and extended light curing. Dent Mater. 2015;31(3):293301.

15. Caneppele TMF, Bresciani E. Resinas Bulk-Fill - O estado da arte. Rev Assoc Paul Cir Dent. 2016;70(3):242-8.

16. Van Ende A, De Munck J, Lise DP, Van Meerbeek B. Bulk-Fill Composites: A Review of the Current Literature. J Adhes Dent. 2017;19(2):95-109.

17. Ivoclar Vivadent. Documentación Científica Tetric N-Ceram Bulk Fill. [Consultado el 13 de enero de 2020]. Accesible en: https://www.ivoclarvivadent.co/es$\mathrm{co} / \mathrm{p} /$ todos/composites/tetric-n-ceram-bulk-fill

18. Pelissier B, Jacquot B, Palin WM, Shortall AC. Three generations of LED lights and clinical implications for optimizing their use. 1: from past to present. Dent Update. 2011;38(10):660-670.

19. AlShaafi MM. Factors affecting polymerization of resin-based composites: A literature review. Saudi Dent J. 2017;29(2):48-58.

20. Gan JK, Yap AU, Cheong JW, Arista N, Tan C. Bulk-Fill Composites: Effectiveness of cure with polyand monowave curing lights and modes. Oper Dent. 2018;43(2):136-43.
21. International Organization for Standardization. ISO 4049:2019. Dentistry - Polymer-based restorative materials. Geneva, Switzerland, 2019.

22. Leprince JG, Leveque P, Nysten B, Gallez B, Devaux J, Leloup G. New insight into the "depth of cure" of dimethacrylate-based dental composites. Dent Mater. 2012;28(5):512-20.

23. Erickson RL, Barkmeier WW. Curing characteristics of a composite. part 2: the effect of curing configuration on depth and distribution of cure. Dent Mater. 2014;30(6):e134-45.

24. Menees TS, Lin CP, Kojic DD, Burgess JO, Lawson NC. Depth of cure of bulk fill composites with monowave and polywave curing lights. Am J Dent. 2015;28(6):357-61.

25. AlShaafi MM, AlQussier A, AlQahtani MQ, Price RB. Effect of mold type and diameter on the depth of cure of three resin-based composites. Oper Dent. 2018;43(5):520-9.

26. Rocha MG, de Oliveira D, Correa IC, Correr-Sobrinho L, Sinhoreti M, Ferracane JL, et al. Light-emitting Diode Beam Profile and Spectral Output Influence on the Degree of Conversion of Bulk Fill Composites. Oper Dent. 2017;42(4):418-27.

27. Moszner N, Zeuner F, Lamparth I, Fischer UK. Benzoylgermanium derivatives as novel visible-light photoinitiators for dental composites. Macromol Mater Eng. 2009;294:877-86.

28. 3M ESPE. Perfil técnico de Filtek One Bulk Fill Restorative. [Consultado el 13 de enero de 2020]. Accesible en: https://multimedia.3m.com/mws/ media/13176710/3m-filtek-one-bulk-fill-restorativetechnical-product-profile.pdf

29. FGM. Instrucciones de uso de Opus Bulk Fill APS. [Consultado el 13 de enero de 2020]. Accesible en: https://www.fgm.ind.br/wp-content/uploads/2019/06/ Manual-opus_bulk_fill.pdf?fbclid=IwAR1MmEDC3LSH8rYck3B3wHQ2lc4XXABil3Uj9WbMObGektxuLQ9zWoHXXFM

30. FGM News 2018 Aesthetics: pág. 24-29 [Consultado el 13 de enero de 2020]. Accesible en: https://issuu.com/ fgmprodutosodontologicos/docs/revista_internaciona1_2018-compress 